\section{P-92 BUILDING THE EVIDENCE BASE FOR COMPLEMENTARY THERAPY IN HOSPICES}

Lara Cowley, Anna Kent, Angeliki Panteli, Amanda Young, Andrea Dechamps. St Wilfrid's Hospice, Eastbourne, UK

\subsection{6/bmjspcare-2017-hospice.119}

Background Complementary Therapy (CT) is widely used in hospices; however the backing research on its efficacy has been somewhat limited. CT can be understood as the bridge between the medical model and alternative therapies. Yet this profession has struggled to gain respect in modern medicine and it is debatable whether Complementary Therapists are seen as equal to other MDT members. Against this background a team of hospice Complementary Therapists have been gathering evidence of their clinical input with a view to assessing and demonstrating its efficacy.

Aims To conduct a wide scale evaluation of CT treatments provided across all services to assess the impact on patient care and for a range of symptoms. Results to guide future evidence based treatment management (the right treatment at the right time).

Methods Patients, using an adapted visual analogue scale, rated their symptoms pre and post CT input. Results, separated into symptom categories, were analysed over a three year period.

Results 1321 patient reported outcomes were collected. After data cleansing, 1217 treatment episodes were reviewed

On average, patient symptoms improved by 2.13 on the 10 point adapted VAS scale which is statistically significant

Pain, breathlessness, anxiety and nausea gained the highest score and were perceived to have the most benefit by patients

Constipation, fatigue, insomnia and appetite had very poor outcomes.

Conclusions We believe the above results demonstrate that CT can indeed provide statistically significant results in a palliative setting. Of particular interest has been the emerging evidence with regards to particular efficacy for particular symptoms. These results can guide other clinicians when referring to the CT team more appropriately therefore meaning patients receive the most suitable treatment for their needs. Such evidence helps with improving the recognition of the role of Complementary Therapy in the hospice setting.

\section{P-93 SPECIALIST PALLIATIVE CARE INPUT IN PATIENTS WITH PARKINSON'S DISEASE}

Rebecca Owen, Jason Ward. St Gemma's Hospice, Leeds, UK

\subsection{6/bmjspcare-2017-hospice. 120}

Background Parkinson's disease (PD) is a common, chronic, progressive neurological condition (NICE, 2006). Its prevalence rate in 2009 was calculated as $27.4 / 10,000$ and is predicted to increase by $28 \%$ by 2020 (Parkinson's UK). NICE guidance for PD recommend that palliative care should be considered throughout all phases of the illness (NICE, 2006).

Methodology The audit reviewed all patients over a three-year period who were referred to St Gemma's Palliative Care Services between October 2013 and October 2016. The computer database SystmOne was used to capture patients who had a coded diagnosis of PD.
Results A total of 38 patients were referred to Palliative Care Services, of whom 20 were still alive at point of collection. There were equal numbers of male and female patients referred and the mean age at referral was 78 years. The mean average documented time from diagnosis to death was 8 years. The average time from referral to death was 6.419 months. However, to specifically focus on the 18 patients who died, eight were referred to palliative care services within the last one month of life and four were referred within the last week. The most common referral reasons to palliative care services were request for help with advance care planning (ACP), marked decline in physical function and dysphagia. Of the patients that were referred, 76\% (29/38) had documentation of ACP and 63\% (24/38) had documentation of DNACPR decision and Gold Standard Framework recommendations. $15 / 18$ patients of those who died had documentation of ACP.

Discussion Patients with PD are much more likely to be referred to specialist palliative care services in the last phases of their illnesses despite NICE guidance. Despite good working relationships with the PD Nurse Specialists, the subgroup of patients referred is a much smaller cohort in comparison to the incidence of PD throughout Leeds.

\section{P-94 ABSTRACT WITHDRAWN}

\section{P-95 TAKING THE RIGHT STEPS TO THE MANAGEMENT OF DELIRIUM AND AGITATION}

Paula Powell, Daniel Monnery, Susan Schofield. Willowbrook Hospice, Merseyside UK

\subsection{6/bmjspcare-2017-hospice. 121}

In 2016 The Cheshire and Merseyside Palliative and End of Life Care Audit Group conducted an audit of the management of delirium in palliative care patients across settings. Willowbrook Hospice (a 12-bedded specialist palliative care unit) took part in this audit. The audit found that benzodiazepines were often used first line for the management of delirium which is not supported by the evidence from the literature. This was felt to be because some staff do not distinguish between agitation and delirium in the dying person. The audit group produced guidelines for the recognition, assessment and management of delirium and recommended the use of the Confusion Assessment Method as an assessment tool.

In response Willowbrook have developed a THINK DELIRIUM policy and accompanying Quick Reference Guide which takes a stepwise approach (similar to the WHO analgesic ladder) to delirium and agitation recognition, assessment and management. In addition we developed a teaching tool that could be delivered easily $1: 1$ or at daily handovers. All staff were updated over a period of a few weeks. Staff now report increased understanding of the differences between delirium and agitation and confidence in management. We are planning a second audit over the next few months.

This poster describes the THINK DELIRIUM project and the stepped approach to management including the outcome of further audit and a survey of the confidence of staff in 